\title{
Role of Hypoxia-Inducible Factors in Acute Kidney Injury
}

\author{
Kelly K. Andringa ${ }^{a}$ Anupam Agarwal ${ }^{a, b}$ \\ a Division of Nephrology, Department of Medicine, Nephrology Research and Training Center, Center for \\ Free Radical Biology, University of Alabama at Birmingham, and ${ }^{b}$ Department of Veterans Affairs Medical Center, \\ Birmingham, Ala., USA
}

\section{Key Words}

Acute kidney injury · Hypoxia · Hypoxia-inducible factor · von Hippel-Lindau proteins

\begin{abstract}
Oxygen is vital to mammalian survival. Oxygen deprivation, defined as hypoxia, elicits adaptive responses in cells and tissues, a process regulated by proteins known as hypoxia-inducible factors (HIF). Animal studies have provided compelling data to demonstrate a pivotal role for the HIF pathway in the pathogenesis of acute kidney injury (AKI) that have led to initial human clinical trials examining this pathway in ischemia-reperfusion injury in various organ systems, including the kidney. HIF are master regulators and mediate adaptive responses to low oxygen in tissues and cells. This review will summarize recent key advances in the field highlighting preclinical and clinical studies relevant to the HIF pathway in the pathophysiology of AKI.
\end{abstract}

(c) 2014 S. Karger AG, Basel

\section{Hypoxia}

Oxygen is vital to the survival of mammalian cells and tissues. Deprivation of adequate oxygen supply, defined as hypoxia, is a critical pathophysiologic event in the pathogenesis of acute kidney injury (AKI). Hypoxia can occur systemically but most generally it is localized to a particular location or tissue [1]. Hypoxia is not only critical in cardiopulmonary conditions but also in the regulation of the microenvironment of every cell $[1,2]$. Understanding the molecular regulation of hypoxia and how cells and organisms adapt to hypoxia is a major area of research focus. The key proteins that regulate such adaptive tissue and cellular responses to low oxygen levels are hypoxia-inducible factors (HIF).

\section{Hypoxia-Inducible Factors}

HIF-1 and HIF-2 are a family of heterodimeric basic helix-loop-helix transcription factors that belong to the Per-Arnt-Sim (PAS) family of regulators [3]. These basic helix-loop-helix domains mediate dimerization and bind to contact DNA of target genes and activate transcription [1]. HIF have an oxygen-sensitive a subunit and a constitutively expressed $\beta$ subunit [2]. Transcriptional regulation of HIF occurs when the HIF heterodimer binds to a hypoxia response element [2]. They are present on regulatory regions of hypoxia-responsive genes [2]. HIF-1 and

Targeting Recovery from Acute Kidney Injury: Round Table Conference at the 19th International Conference on Continuous Renal Replacement Therapies (Manchester Grand Hyatt, San Diego, Calif., USA, March 2-3, 2014).

\section{KARGER}

E-Mail karger@karger.com www.karger.com/nec
(C) 2014 S. Karger AG, Basel

1660-2110/14/1274-0070\$39.50/0
Anupam Agarwal, MD

University of Alabama at Birmingham

1900 University Boulevard, THT647

Birmingham, AL 35294 (USA)

E-Mail agarwal@uab.edu 
HIF-2 have distinct functions that only minimally overlap [2]. The gene targets for HIF-1 and HIF-2 are not completely clear but may involve interactions with nuclear factors and cofactors [2]. The HIFa subunit is the regulator of HIF activation, when it is allowed to translocate to the nucleus where it dimerizes with the $\beta$ subunit and recruits transcriptional cofactors, such as p300 and others [2]. HIF-1a mRNA is expressed in almost all tissues in humans and rodents [1]. HIF-2 $\alpha$ shows a more selective expression pattern in tissues and is largely localized to the endothelium and in interstitial cells of the kidney [2]. HIF levels are regulated by the von Hippel-Lindau (VHL) proteins (pVHL).

\section{Von Hippel-Lindau Proteins}

pVHL is a tumor suppressor protein which is part of the E3 ubiquitin ligase complex that targets HIFa for degradation [2]. pVHL is the gene product and is critical for targeting the a portion of HIF for proteolysis [2]. Under normoxic conditions, HIFa binds to VHL and then the prolyl hydroxylase domain (PHD)-containing proteins recruit ubiquitin to help degrade the complex [2]. There are three PHDs, PHD1, PHD2 and PHD3, which display tissue-specific expression patterns. All three PHDs are found to be expressed in renal epithelial cells [2]. Pharmacological inhibition of PHDs has been used to stimulate HIF-dependent genes, such as erythropoietin (EPO), to help in improving the outcome of ischemic injury in animal models [3]. During hypoxia, however, prolyl hydroxylases are inactivated and HIFa degradation does not occur [2]. This leads to an accumulation and stabilization of HIFa, which is then translocated into the nucleus, binding the $\alpha$ subunit to the $\beta$ subunit and to cognate DNA binding sequences and transcription of target genes [2]. In renal carcinoma, cell lines that lack the expression of VHL, HIF- $1 \alpha$ and HIF- $2 \alpha$ are both maximally activated. This expression can be restored in cells that are transfected with VHL expression vectors [1], thereby, implicating a critical role of both VHL and HIF in the ability to regulate and coordinate the cellular response to hypoxic conditions.

\section{Regulators}

Besides hypoxia, several other mediators can regulate HIF. These include reactive oxygen species, generated by mitochondrial complex III, nitric oxide, cobalt chloride, tumor necrosis factor- $\alpha$, angiotensin II and others [2]. HIF is involved in the regulation of many biological processes related to kidney function, such as glucose and energy metabolism, angiogenesis, erythropoiesis, and iron homeostasis, cell migration, vasomotor regulation, and cell-cell and cell-matrix interactions [2]. There are a number of HIF-regulated genes, and the list continues to grow as more mechanisms are elucidated [2]. These include vascular endothelial growth factor (VEGF), heme oxygenase-1 (HO-1), connective tissue growth factor, EPO and others [2]. Figure 1 shows examples of direct transcriptional HIF targets with relevance to kidney disease [2].

\section{Animal Studies}

Tissue hypoxia is a normal part of embryogenesis, implying an important role for HIF in development [2]. It is not completely understood how HIF and VHL are required in kidney development, but they exhibit cell- and stage-specific patterns during nephrogenesis [2]. In animal models, inactivation of HIF- $1 \alpha$, HIF- $2 \alpha$ or VHL in germ cell lines results in either embryonic or perinatal lethality [2]. In mice that do not express pVHL, embryonic death occurs during mid gestation and primarily due to abnormal vascularization of the placenta [3]. This phenotype appears to be mediated by HIF [3]. In mouse models, it has been shown that inhibition of HIFa degradation leads to improved EPO levels [2]. This inhibition also improves iron uptake, while also suppressing hepatic hepcidin. Hepcidin inhibits iron transport and is a key factor in the development of anemia.

Rats exposed to hypoxic conditions $\left(7 \% \mathrm{O}_{2}\right)$ show that HIF-1 $\alpha$ and HIF-2 $\alpha$ mRNA are detected in the brain, heart, kidney, liver, lung, and spleen, while C57BL/6J mice under hypoxic conditions show a more pronounced induction in the brain, kidney, and lung [4]. In a mouse model (C57BL/6J) of AKI, Kapitsinou et al. [5] tested pretreatment with a prolyl hydroxylase inhibitor, GSK1002083A, before an ischemic event. In this preconditioning protocol, animals were treated with the inhibitor or vehicle control before clamping of the renal pedicle. The authors showed that pretreatment before the ischemic event preserved kidney function and prevented the development of fibrosis, inflammation, and anemia. In contrast, inhibiting HIF prolyl hydroxylation during the recovery phase (postischemic event) did not have a protective effect [5]. 


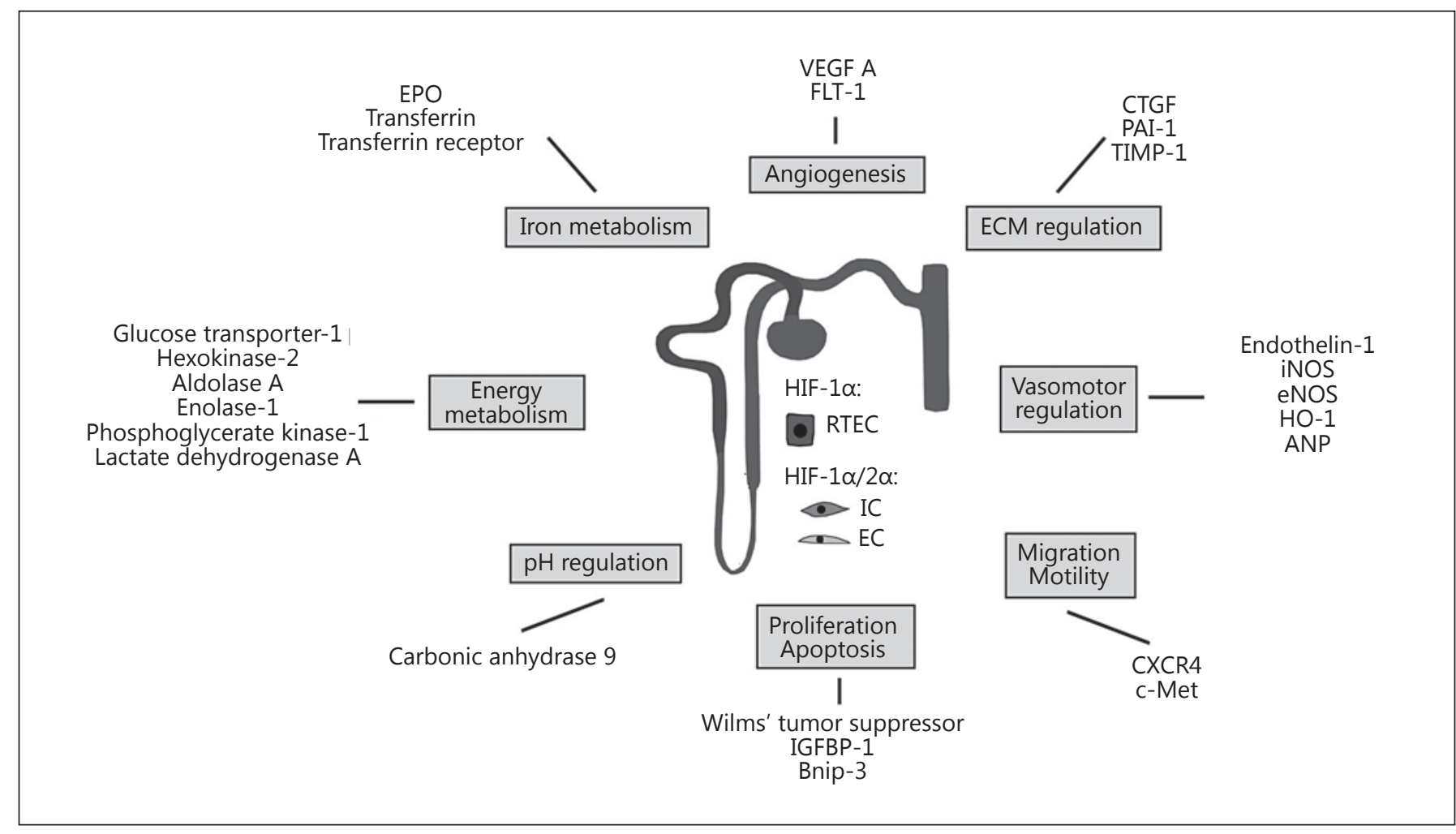

Fig. 1. Examples of direct transcriptional HIF targets with relevance to kidney function. Shown are selected direct HIF target genes and their classification into functional groups. For a comprehensive list of HIF target genes, the reader is referred to Wenger et al. [14]. Some of the HIF targets listed here appear to be preferentially regulated by HIF-2 (e.g. VEGF and EPO). In contrast to HIF-2 $\alpha$, HIF- $1 \alpha$ is expressed in most renal epithelial cells, whereas HIF- $2 \alpha$ is mainly found in endothelial cells and renal interstitial fibroblast-like cells. HIF-1 $\alpha$ is also expressed in papillary and inner medullary interstitial and endothelial cells but was not detected in interstitial an endothelial cells of the cortex and outer medulla. $\mathrm{ANP}=$ Atrial natriuretic peptide; Bnip-3 = BCL2/adenovirus E1B

In another study, the authors, also interested in the outcomes of treatment for AKI, examined 2-oxoglutarate analogues [6]. Since oxoglutarate is a substrate of PHD, analogues are used to inhibit PHD and led to stabilization of HIF in the presence of oxygen. Pretreatment with 2-oxoglutarate analogues has demonstrated preservation of kidney function and structure in the presence of acute ischemic injury [6]. The authors used a mouse model and induced ischemia-reperfusion injury in the kidney and treated with two 2-oxoglutarate analogues, dimethyloxalyl glycine and oxalyl glycine. The results showed that HIF-1 a expression was increased and confined to the kidneys, especially in proximal tubule cells, and afforded a protective effect. Using medicinal chemistry approaches,
19-kDa-interacting protein 3 (proapoptotic $\mathrm{BH} 3$ domain; only BCL-2 family member); $\mathrm{c}$-Met = tyrosine kinase receptor for scat ter factor/hepatocyte growth factor; CTGF $=$ connective tissue growth factor; CXCR4 = chemokine receptor 4; EC = endothelial cell; $\mathrm{ECM}=$ extracellular matrix; eNOS = endothelial nitric oxide synthase; FLT-1 = fetal liver tyrosine kinase- 1 (VEGF receptor-1); IC = interstitial cell; IGFBP-1 = insulin-like growth factor binding protein-1; iNOS = inducible nitric oxide synthase; PAI-1 = plasminogen activator inhibitor-1; RTEC = renal tubular epithelial cell; TIMP- $1=$ tissue inhibitor of metalloproteinase- 1 . This figure is reprinted with permission from the American Physiological Society from Haase [2].

they synthesized two compounds with enhanced pharmacokinetic profiles and showed that one of these molecules, 2-(1-chloro 4-hydroxy isoquinoline-3-carboxamido) acetic acid, was protective in AKI. This result may offer a feasible target for treatment in AKI from ischemic events [6].

Acetazolamide is commonly prescribed to treat glaucoma and high-altitude mountain sickness. It has been reported to induce vessel dilation (including in the renal system) and is also a potent inducer of HIF-1 $\alpha$ [7]. In a mouse model of ischemia-reperfusion injury, treatment with acetazolamide improved renal function by decreasing creatinine and plasma blood urea nitrogen compared to the vehicle-treated animals [7]. In cell culture, using 
HK-2 cells, treatment with acetazolamide showed a cytoprotective effect even after ischemic injury [7]. These studies show how acetazolamide may be effective in renoprotection in AKI.

In rat renal medullary interstitial cells, hypoxia causes a significant increase in HO-1 mRNA and inhibitors of HIF-1 $\alpha$ degradation reverse such activation of HO-1 [reviewed in ref. 8]. HIF-1 has been shown to regulate hypoxia-mediated HO-1 induction in rat aortic vascular cells but not in pulmonary cells; this difference implies that regulation of genes to hypoxia differs in cells from various systems. It is also important to note that in the case of $\mathrm{HO}-1$, hypoxia is a potent activator in rodents, but most human cells/tissues show hypoxia-mediated HO-1 repression [reviewed in ref. 8]. Figure 2 depicts the role of HIF activation in kidney disease. Both ischemic and nonischemic AKI leads to renal hypoxia thus activating HIF [9]. Once HIF is activated, it protects the kidney against hypoxia and induces adaptive responses [9].

\section{Clinical Studies}

While animal models have led to novel targets and a better understanding on how AKI occurs, the use of pharmaceuticals is still relatively untested in human AKI. As noted above, there are differences in the regulation of genes between rodents and human. A current phase II study in which the investigators are testing the hypothesis that GSK1278863 (a HIF prolyl hydroxylase inhibitor) will reduce neurologic, renal, and/or cardiac ischemia in patients undergoing elective descending thoracic aorta/thoracoabdominal aortic aneurysm repair (NCT01920594 [10]). At the time of writing this review, this study was not yet actively recruiting patients. In a currently active study, GSK1278863 is being tested in patients with renal impairment (including patients on hemodialysis) in comparison to healthy individuals (NCT01406340 [11]). This study is testing the pharmacokinetics of GSK1278863 and will likely help direct future dosing paradigms.

\section{Evidence from Evolution}

High-altitude Tibetans are able to maintain normal aerobic metabolism despite profound arterial hypoxia, while visitors to this region demonstrate increased hemoglobin levels at this same altitude [12]. In these high-altitude communities, adaptations occur, as can be seen in the birth weight, hemoglobin levels, oxygen saturation in the blood

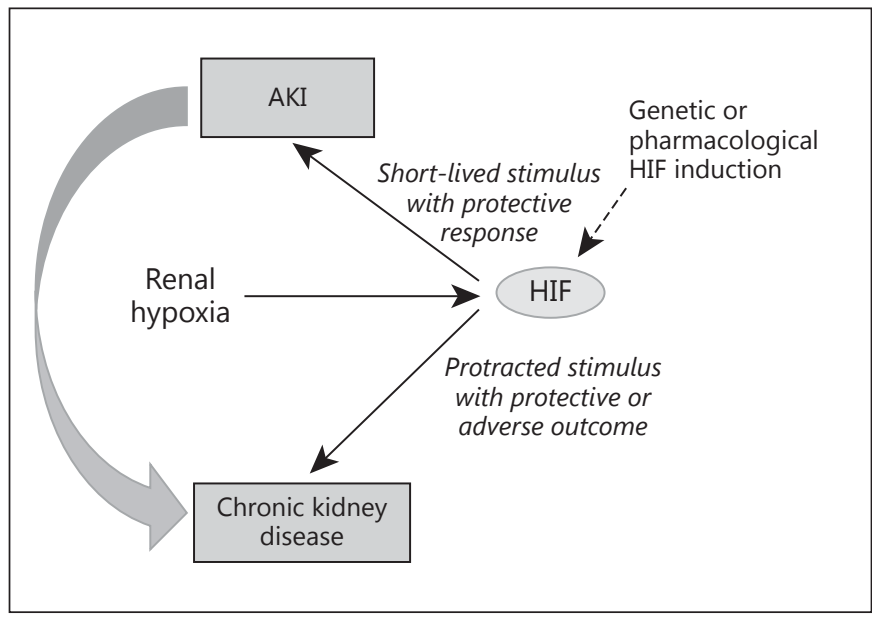

Fig. 2. HIF activation in kidney disease. Both ischemic and nonischemic AKI leads to hypoxia of the kidney, which, in turn, activates HIF. Activated HIF protects the kidney against hypoxia and induces adaptive responses. Chronic kidney disease and hypoxia of the kidney compose a vicious cycle. Hypoxia activates HIF, which protects the kidney against tissue injury due to oxygen depletion. Furthermore, the kidney suffering from chronic kidney disease is paradoxically protected against AKI under some conditions. This paradoxical tolerance may be mediated by HIF activation as hypoxic preconditioning. However, HIF activation may also result in adverse outcomes of the disease under certain conditions. This figure is reprinted with permission from Wiley Publishing from Nangaku et al. [9].

of infants, and in adults after exercise [13]. One of the variations found in these genetic studies on high-altitude Tibetans versus their lower-altitude Asian counterparts was a variation in EGLN1, also known as Egl nine homolog 1, and a gene that encodes PHD2. EGLN1 targets two HIF-1a proteins for degradation under normoxic condition decreasing the transcription of HIF targets (like EPO). These changes in EGLN1 in the high-altitude genome might imply that HIF and HIF targets are important in this adaptation to high altitude seen in this population [12]. Another variation was in the EPAS1 gene, which is part of the PAS protein family and is also known as HIF-2 $\alpha$ [13]. Persons with this EPAS1 variant demonstrate lower erythrocyte levels and hemoglobin levels [13].

\section{Conclusion}

HIF is a master regulator switch for the adaptive response to cellular and tissue hypoxia. Modification of HIF is protective in multiple animal models of AKI, and clinical studies to explore these effects in humans are ongoing 
and of great interest. There is a cell-specific distribution of HIF-1 and HIF-2 and the regulation of these genes is tissue specific. There are also species-specific effects on responses to hypoxia. What works in rodents might not always be translatable to humans. Learning how specific tissues respond to hypoxic events will help in narrowing down therapeutic regimens to better prevent AKI and hasten recovery. Hypoxia is associated with the recovery phase of AKI and further mechanistic studies will help elucidate the effects of these hypoxic events on the tissue recovery and repair. HIF activation shows a protective effect in the setting of AKI but has a controversial role in the pathogenesis of chronic kidney disease, needing further investigative efforts.

\section{Acknowledgments}

The study was supported by NIH grants R01 DK059600 (A.A.) and O’Brien Center P30 DK079337 (A.A.).

\section{Disclosure Statement}

The authors have no conflicting financial interests.

\section{References}

1 Semenza GL: HIF-1: mediator of physiological and pathophysiological responses to hypoxia. J Appl Physiol 2000;88:1474-1480.

$\checkmark 2$ Haase VH: Hypoxia-inducible factors in the kidney. Am J Physiol Renal Physiol 2006; 291:F271-F281.

3 Haase VH: The VHL tumor suppressor: master regulator of HIF. Curr Pharm Des 2009; 15 3895-3903.

-4 Semenza GL, Agani F, Booth G, Forsythe J, Iyer $\mathrm{N}$, Jiang $\mathrm{BH}$, Leung $\mathrm{S}$, Roe $\mathrm{R}$, Wiener $\mathrm{C}$, Yu A: Structural and functional analysis of hypoxia-inducible factor 1 . Kidney Int 1997; 51:553-555.

5 Kapitsinou PP, Jaffe J, Michael M, Swan CE, Duffy KJ, Erickson-Miller CL, Haase VH: Preischemic targeting of HIF prolyl hydroxylation inhibits fibrosis associated with acute kidney injury. Am J Physiol Renal Physiol 2012;302:F1172-F1179.

6 Schley G, Klanke B, Schodel J, Kroning S, Turkogly G, Beyer A, Hagos Y, Amann K, Burckhardt BC, Burzlaff N, Eckardt KU, Willam C: Selective stabilization of HIF1a in renal tubular cells by 2-oxyglutarate analogues. Am J Pathol 2012;181:1595-1606.
7 An Y, Zhang JZ, Han J, Yang HP, Tie L, Yang XY, Xiaokaiti Y, Pan Y, Li XJ: Hypoxia-inducible factor-1a dependent pathways mediate the renoprotective role of acetazolamide against renal ischemia-reperfusion injury. Cell Physiol Biochem 2013;32:1151-1166.

-8 Sikorski EM, Hock T, Hill-Kapturczak N, Agarwal A: The story so far: molecular regulation of the heme oxygenase-1 gene in renal injury. Am J Physiol Renal Physiol 2004; 286:F425-F441.

$>9$ Nangaku M, Rosenberger C, Heyman SN, Eckardt KU: Regulation of hypoxia-inducible factor in kidney disease (review). Clin Exp Pharmacol Physiol 2013;40:148-157.

10 Clinical Trials Government website Identifier NCT01920594: Study of GSK1278863 to reduce ischemic events in patients undergoing thoracic aortic aneurysm repair. http://clinicaltrials.gov/ct2/show/NCT01920594?term= NCT01920594\&rank=1.

11 Clinical Trials Government website Identifier NCT01406340: Assessment of the pharmacokinetics of GSK1278863 and metabolites in normal subjects and subjects with renal impairment. http://clinicaltrials.gov/ct2/show/ NCT01406340.
2 Simonson TS, Yang Y, Huff CD, Yun H, Qin G, Witherspoon DJ, Bai Z, Lorenzo FR, Xing J, Jorde LB, Prchal JT, Ge RL: Genetic evidence for high-altitude adaptation in Tibet. Science 2010;329:72-74

13 Yi X, Liang Y, Huerta-Sanchez E, Jin X, Cuo ZX, Pool JE, Xu X, Jiang H, Vinckenbosch N, Korneliussen TS, Zheng H, Liu T, He W, Li K, Luo R, Nie X, Wu H, Zhao M, Cao H, Zou J, Shan Y, Li S, Yang Q, Asan, Ni P, Tian G, Xu J, Liu X, Jiang T, Wu R, Zhou G, Tang M, Qin J, Wang T, Feng S, Li G, Huasang, Luosang J, Wang W, Chen F, Wang Y, Zheng X, Li Z, Bianba Z, Yang G, Wang X, Tang S, Gao G, Chen Y, Luo Z, Gusang L, Cao Z, Zhang Q, Ouyang W, Ren X, Liang H, Zheng H, Huang Y, Li J, Bolund L, Kristiansen K, Li Y, Zhang Y, Zhang X, Li R, Li S, Yang H, Nielsen R, Wang J, Wang J: Sequencing of 50 human exomes reveals adaptation to high altitude. Science 2010;329:75-77.

14 Wenger RH, Stiehl DP, Camenisch G: Integration of oxygen signaling at the consensus HRE. Sci STKE 2005;2005:re12. 